\title{
Comparison of two digital intraoral radiography imaging systems as a function of contrast resolution and exposure time
}

\author{
Aydin, Kader C ; Demirel, Oğuzhan ; Özcan, Mutlu
}

\begin{abstract}
BACKGROUND To compare the image quality of two different digital imaging systems; one photostimulable phosphor plate system (PSP) and a direct digital radiography system with CMOS imaging sensor; via evaluating contrast resolution among four different exposure times. METHODS Endodontically treated incisor teeth embedded in paraffin blocks are aligned next to a 99.5\% Al wedge and exposed for 0.8, 0.1,0.125 and 0.16 seconds using both the CMOS and PSP systems. Using ImageJ software, 5 isometric and isogridded ROI from each root filling area and isometric ROI from the $\mathrm{Al}$ stepwedge were calculated. RESULTS Evaluation of the total of 120 images displayed that PSP system produced significantly higher contrast resolution $(\mathrm{P}<0.05)$ in regard to pixel values than the CMOS. The CMOS system was non- responsive to increasing dose $(\mathrm{P}=0.000)$. Regarding the EqAl values, no significant difference was determined between groups $(\mathrm{P}>0.05)$. CONCLUSIONS The contrast resolution was higher using the PSP system. It can be estimated that, filling material will be more obvious under lower doses using PSP.
\end{abstract}

DOI: https://doi.org/10.23736/S0026-4970.19.04286-9

Posted at the Zurich Open Repository and Archive, University of Zurich

ZORA URL: https://doi.org/10.5167/uzh-197138

Journal Article

Accepted Version

Originally published at:

Aydin, Kader C; Demirel, Oğuzhan; Özcan, Mutlu (2020). Comparison of two digital intraoral radiography imaging systems as a function of contrast resolution and exposure time. Minerva Stomatologica, 69(3):148-152.

DOI: https://doi.org/10.23736/S0026-4970.19.04286-9 
Comparison of Two Digital Intraoral Radiography Imaging Systems as a Function of Contrast Resolution and Exposure Time

Kader Cesur Aydin ${ }^{1^{*}}$, Oğuzhan Demirel ${ }^{2}$, Mutlu Özcan $^{3}$.

${ }^{1^{*}}$ Assist. Prof. Dr., Department of Dentomaxillofacial Radiology, School of Dentistry, Istanbul Medipol University, Istanbul, Turkey. kadercesur@yahoo.com

${ }^{2}$ Assist. Prof. Dr., Bahçeşehir University, Department of Dentomaxillofacial Radiology, School of Dentistry, Istanbul, Turkey. dtoguzhandemirel@gmail.com

${ }^{3}$ Prof. Dr., University of Zürich, Dental Materials Unit, Center for Dental and Oral Medicine, Clinic for Fixed and Removable Prosthodontics and Dental Materials Science, Zürich, Switzerland mutluozcan@hotmail.com

${ }^{*}$ Corresponding Author: Kader Cesur Aydın

Department of Dentomaxillofacial Radiology, School of Dentistry, Istanbul Medipol University, Istanbul, Turkey, Istanbul, Turkey. kadercesur@yahoo.com 


\section{ABSTRACT}

Background To compare the image quality of two different digital imaging systems; one photostimulable phosphor plate system (PSP) and a direct digital radiography system with CMOS imaging sensor; via evaluating contrast resolution among four different exposure times. Material and Methods Endodontically treated incisor teeth embedded in paraffin blocks are aligned next to a $99.5 \%$ Al wedge and exposed for 0.8, 0.1,0.125 and 0.16 seconds using both the CMOS and PSP systems. Using Image J software, 5 isometric and isogridded ROI from each root filling area and isometric ROI from the Al stepwedge were calculated. Results Evaluation of the total of 120 images displayed that PSP system produced significantly higher contrast resolution $(p<0.05)$ in regard to pixel values than the CMOS. The CMOS system was non- responsive to increasing dose $(p=0.000)$. Regarding the EqAl values, no significant difference was determined between groups $(p>0.05)$. Conclusions The contrast resolution was higher using the PSP system. It can be estimated that, filling material will be more obvious under lower doses using PSP.

Key Words: Dental Radiography- Radiologic Technology- Diagnostic Equipment 


\section{Introduction}

Digital imaging systems have gained high-rate acceptance in comparison to conventional radiographies in dentistry due to increase in diagnostic capabilities and even capacity of virtual planning. Depending on the type of the image acquisition system properties, dental digital radiography systems may be classified in two major groups; direct digital radiography systems with flat panel detectors (CCD, CMOS, IRFPA) and photostimulable phosphor plate systems (PSP). Initial meeting of the dental society with digital systems has been via direct digital system namely RadioVisioGraphy (Trophy Radiologie, Vincennes, France) in 1989. ${ }^{1-5}$ The evolution of photostimulable phosphor plate systems (PSP) became later on. ${ }^{6}$

Working either experimentally or in the clinic, these two systems have several opportunities or disadvantages compared to each other. To make decisions on which system to choose for daily clinical usage, the most important data comes from two radiation optimization strategies;

1. Application of the ALARA principle (As Low As Reasonably Achievable; to prevent unnecessary radiation and overexposure; minimum time, optimum distance and optimum shielding) to diminish the patient dose

2. Improving visual quality, that means contrast resolution for the systems. ${ }^{7}$

The aim of this study is to compare the image quality of two different digital imaging systems; one photostimulable phosphor plate system (PSP) with a direct digital radiography system with CMOS imaging sensor; via evaluating contrast resolution among four different irradiation tracts.

\section{Materials and Methods}


One photostimulable phosphor plate system (PSP) (Sopro S.A. , ACTEON Group, La Ciotat, France) and one direct digital radiography system with CMOS imaging sensor (Kodak 5100, Carestream Dental, Atlanta, Canada.) are used with similar size sensors (Number 1) for comparison of the contrast resolution. Technical specifications of the two systems are shown in Table 1. Endodontically treated single rooted incisor teeth embedded in paraffin blocks are used as shooting materials. A 10 stepped $99.5 \%$ aluminum wedge with uniform $1 \mathrm{~mm}$ steps is included in all shootings for comparison of the contrast resolution in equivalance (Picture 1). 15 models involving a total number of 43 teeth were exposed both for the CMOS and PSP groups at 4 different exposure times. Total number of images evaluated was 120. The standard geometric configuration for the x-ray source-object distance was set at $30 \mathrm{~cm}$, with zero degrees horizontal and vertical angulations of the $\mathrm{x}$-ray beam. All x- ray shootings were performed by CS 2200 (Carestream Health, Inc. 150 Verona Street Rochester, NY 14 608, USA) operating with $60 \mathrm{kV}$ and $7 \mathrm{~mA}$ electric power supply. For both groups $0.8,0.1,0.125$ and 0.160 seconds of irradiation were performed. All PSP images were immediately scanned via PSPIX imaging plate scanner (ACTEON Group, La Ciotat, France). Images from both systems including 4 different irradiation tracts (total of 344) were transferred and analyzed in a personal computer. Using Image ${ }^{8}$ software, all images obtained from the PSP and CMOS systems were converted to JPEG, gridded and 5 isometric areas including each tooth of each image are calculated as contrast resolution and this procedure is repeated for $0.8,0.1,0.125$ and 0.160 second shootings. Aluminum wedge calculations were performed via isometric area calculations of every step thickness of the wedge for each image. For the evaluation of the findings obtained in this study, IBM SPSS 
Statistics 22 (IBM SPSS, Turkey) program was used. Normal distribution of the study data parameters was evaluated via Shapiro Wilks test and it was determined that the normal distribution was appropriate for the parameters. Two-way ANOVA test was used for determination of the quantitative data; to evaluate the common effect between two imaging groups and different irradiation times. One-way Anova test (post hoc Tukey HSD test) and Student t test were used as follow-up tests. Fisher Freeman Halton Test was used for comparison of qualitative data. Significance was assessed at $p<0.05$ level.

\section{Results}

Two-way ANOVA test results revealed significant differences among CMOS and PSP groups $(p=0.000 ; p<0.05)$. Evaluation of different time-interval shooting tracts also revealed significant differences $(p=0.000 ; p<0.05)$. The common effect of groups and shooting tracts revealed significant differences as well $(p=0.000 ; p<0.05)$. For evaluation of groups in different time-interval shooting tracts, Student $t$ test and Oneway ANOVA test was performed. Mean radiopacity values of CMOS and PSP groups in shooting tracts are presented in Table 2. PSP system revealed more radiolucent results in comparison with the CMOS system in all four shooting tracts. For all 0.08 , $0.1,0.125$ sec tracts $(p=0.000 ; p<0.05)$ and 0.16 sec tract $(p=0.009 ; p<0.05)$ PSP group values were significantly higher than the CMOS group. Intragroup evaluation for CMOS group values showed no significance between time tracts $(p=0.872$; $p>0.05)$. Intragroup evaluation for PSP group values displayed that there is statistically significant difference between the measurement averages for $0.08,0.1$, 0.125 and $0.16 \mathrm{sec}$ of irradiation. As a result of the binary comparisons performed to determine the dose from which the significance was derived; the measurement 
average of $0.08 \mathrm{sec}$ irradiation was significantly higher than the $0.125(p=0.000)$ and 0.16 sec doses $(p=0.000 ; p<0.05)$ respectively, while the mean of 0.1 sec irradiation was not significantly different $(p>0.05)$. The measurement average of 0.1 sec shooting tract was significantly higher than $0.125(p=0.000)$ and $0.16 \mathrm{sec}(p: 0.000)$ irradiation $(p<0.05)$. The average of $0.125 \mathrm{sec}$ shooting was found to be significantly higher than the mean of 0.16 sec of irradiation, as well $(p=0.000 ; p<0.05)$. The relation of the calculated isometric areas with the aluminum wedge in different irradiation tracts is determined as the minimum step thickness equivalence (EqAl). For this purpose Fisher Freeman Halton Test is used. The minimum mean isometric area calculations detected from the root canals was equivalent to $5 \mathrm{~mm}$ Al step thickness. For all 4 different irradiation times, CMOS and PSP groups revealed no significant difference in comparison to Al wedge equivalence distributions $(p>0.05)$. Distribution of Al wedge equivalence of the CMOS and PSP groups in $0.8,0.1,0.125$ and $0.16 \mathrm{sec}$ of irradiation is revealed in Table 3 .

\section{Discussion}

Some of the recent experiments about dental image quality, mainly evaluated the images in means of acceptable visual interpretation ${ }^{9-13}$. Classification of visual interpretation may be valuable in clinical enquiries, alas, may cause diversified interpretations in regard to the radiologist qualifications.

To obtain objective dataset, quantitative data about the evaluated specimen may be valuable when the study sample is standardized (distance, dose, collimator size etc.). In this study we evaluated contrast resolution of the images via evaluation through Image ${ }^{8}$ and obtained quantitative data in regard to image quality via contrast resolution. 
Collimator size and shape may alter image contrast, and smaller diameter round collimators may enhance high- contrast image formation. ${ }^{14}$ In this study, single type of rounded collimator $53 \mathrm{~mm}$ in diameter was used, therefore no alteration of the contrast resolution was caused due to the collimator.

Digital imaging systems provide 255 gray shades regarding 0 as black, and these gray values can be used in order to measure exposure. The pixel values among 256 shades are used to determine contrast resolution. ${ }^{15}$ Stamatakis et.al evaluated dose response qualities of Digora PSP and concluded that the dose response is linear with the gray values. ${ }^{16}$ Similar results are obtained for the PSP group in this study, revealing decreased radiopacity with increasing doses, alas, CMOS group revealed non-linear dose response. By the way, it is possible to evaluate conventional radiographs for radioopacity of materials under constant dose and automatic processing procedures. $^{17}$

For determining the radiopacity, the JPEG images were gridded and isometric area measurements that belong to 5 intracanal filling lengths were calculated. Mean calculations were used as contrast resolution of the tooth. Similar techniques were used in detecting radiopacity of different filling materials. ${ }^{18-20}$ Aluminum step- wedge equivalencies analyzed for all doses in groups revealed no significant difference, that is, regardless of the increasing dose EqAl values were similar between groups, all root canal fillings having a value higher than $5 \mathrm{~mm}$ EqAl.

Akcay et al compared conventional graphics, CMOS and PSP images for ability of detecting radiopacity on different root canal sealers, and presented that some sealers have higher radiopacity at either PSP or CMOS images over conventional graphies. ${ }^{21}$ It is also stated in the same study that, all materials had a radiopacity level above 
$3 \mathrm{~mm}$ EqAI, that is stated by the ANSI/ADA ${ }^{22}$ specification 57 and ISO-specification limits. ${ }^{23}$

The main distinctive feature for any radiographic device should be the capacity for obtaining the ALARA principle. ${ }^{24}$ To achieve the maximal image quality at the minimum dose, criteria about exposure settings have been reported at NCRP ${ }^{25,26}$ and ICRP. ${ }^{27,28}$ In evaluation of sensitivity, mean pixel value of the isometric ROI (Region of Interest) pixel vales are evaluated as higher the pixel value, lower the radiopacity. ${ }^{29}$ For this purpose, contrast resolution measurements for different exposure times of CMOS and PSP group evaluations were determined and PSP was found significantly more sensitive for all doses. Doyle and Finney $(2005)^{30}$ and Borg et al $(2000)^{15}$ stated that due to the wider latitude of PSP's over CCD and CMOS sensors, fewer uptakes are needed. Alas, Udupa et al stated this wideness condition as a risk factor for increasing exposure times, that may result in higher irradiation doses. ${ }^{31}$ The image quality is very good both in low and high doses at PSPs, in this case, we presume that having a better diagnostic quality in a lower dose will eventually lead to selection of decreasing exposure times. Regarding the CMOS group, which dos not reveal decreasing radiopacity with increasing exposure times, usage of higher doses due to low diagnostic quality may outcome as a risk.

Regarding dose response and subjective image quality of conventional, CMOS and PSP systems, Bhaskaran et al presented that at lower doses digital systems provided better quality images in comparison to conventional radiographs. As well, it was found that dose reduction ratios in comparison to conventional graphics displayed significant difference for PSP over CMOS. ${ }^{32}$ 


\section{Conclusions}

From this study the following could be concluded:

1) Regarding the CMOS and PSP systems, PSP revealed more radiopacity (contrast resolution) at all doses tested. This data would enlighten wider usage for the clinicians.

2) PSP displayed decreasing radiopacity with increasing doses, alas, CMOS have non significant radiopacity decrease with the increased doses.

3) For further studies, experimental analysis of dose susceptibility upon a wider range of shooting times for PSP/ CMOS can be suggested. 


\section{REFERENCES}

1. Mouyen F, Benz C, Sonnabend Å, Lodter J. Presentation and physical evaluation of RadioVisioGraphy. Oral Surg Oral Med Oral Pathol Oral Radiol 1989;68:238-42.

2. Horner K, Shearer A, Walker A, Wilson N. RadioVisioGraphy: An initial evaluation. Braz Dent J 1990;168:244-8.

3. Walker A, Horner K, Czajka J, Shearer C, Wilson H. Quantitative assessment of a new dental imaging system. Braz J Radiol 1991;64:529-36.

4. Benz C, Mouyen F. Evaluation of the new RadioVisioGraphy system image quality. Oral Surg Oral Med Oral Pathol Oral Radiol 1991;72:627-31.

5. Herford AS, Miller M, Lauritano F, Cervino G, Signorino F, Maiorana C. The use of virtual surgical planning and navigation in the treatment of orbital trauma. Chin J Traumatol. 2017;20(1):9-13. doi: 10.1016/j.cjtee.2016.11.002.

6. Schaetzing R, Whiting BR, Lubinsky AR, Owen JF. Digital radiography using storage phosphors. In: Newell JD, Kelsey CA, eds. Digital imaging in diagnostic radiology. Edinburgh: Churchill Livingstone 1990:107-38.

7. Busch HP, Faulkner K. Image quality and dose management in digital radiography: a new paradigm for optimisation. Rad Prothet Dosimet 2005;117:143-7.

8. Rasband, W.S., Image J, U. S. National Institutes of Health, Bethesda, Maryland, USA, https://imagej.nih.gov/ij/, 1997-2016.

9. de Almeida SM, de Oliveira AE, Ferreira RI, Bóscolo FN. Image quality in digital radiographic systems. Braz Dent J 2003;14:136-41.

10. Farrier SL, Drage NA, Newcombe RG, Hayes SJ, Dummer PM. A comparative 
study of image quality and radiation exposure for dental radiographs produced using a charge-coupled device and a phosphor plate system. Int Endod J 2009;42:900-7.

11. Borg E, Attaelmanan A, Gröndahl HG. Subjective image quality of solid-state and photostimulable phosphor systems for digital intra-oral radiography. Dentomaxillofacial Radiol. 2000;29:70-5.

12. De Melo DP, Cruz AD, Melo SL, De Farias JF, Haiter-Neto F, De Almeida SM. Effect of Different Tube Potential Settings on Caries Detection using PSP Plate and Conventional Film. J Clin Diagnost Res 2015;9:ZC58-61.

13. Yalcinkaya S, Künzel A, Willers R, Thoms M, Becker J. Subjective image quality of digitally filtered radiographs acquired by the Dürr Vistascan system compared with conventional radiographs. Oral Surg Oral Med Oral Pathol Oral Radiol 2006;101:643-51.

14. Falk A, Lindhe JE, Rohlin M, Nilsson M. Effects of collimator size of a dental X-ray unit on image contrast. Dentomaxillofacial Radiol 1999;28:261-6.

15. Borg E, Attaelmanan A, Grondahl HG. Image plate systems differ in physical performance. Oral Surg Oral Med Oral Pathol Oral Radiol 2000;89:118-24.

16. Stamatakis HC, Welander U, McDavid WD. Dose response of a storage phosphor system for intraoral radiography. Dentomaxillofacial Radiol. 1999;28:272-6.

17. Pekkan G, Ozcan M. Radiopacity of different resin-based and conventional luting cements compared to human and bovine teeth. Dental Mater J 2012;31:68-75.

18. Tanomaru-Filho M, Jorge EG, Tanomaru JM, Gonçalves M. Evaluation of the 
radiopacity of calcium hydroxide- and glass-ionomer-based root canal sealers. Int Endod J 2008;41:50-3.

19. Reis JM, Jorge EG, Ribeiro JG, Pinelli LA, Abi-Rached Fde O, TanomaruFilho M. Radiopacity evaluation of contemporary luting cements by digitization of images. ISRN Dent. 2012;2012:704246.

20. Baksi Akdeniz BG, Eyüboglu TF, Sen BH, Erdilek N. The effect of three different sealers on the radiopacity of root fillings in simulated canals. Oral Surg Oral Med Oral Pathol Oral Radiol 2007;103: 138-41.

21. Akcay I, Ilhan B, Dundar N. Comparison of conventional and digital radiography systems with regard to radiopacity of root canal filling materials. Int Endod J 2012;45:730-6.

22. ANSI/ADA (2000) American National Standards Institute/American Dental Association: specification no. 57, endodontic sealing materials. New York: ANSI/ADA.

23. International Organization for Standardization (2001) ISO 6876, dental root canal sealing materials, 2nd edn. Geneva, Switzerland: ISO.

24. Eastman TR. ALARA and digital imaging systems. RadiologTechnol 2013;84:297-8.

25. National Council on Radiation Protection and Measurements.Radiation Protection in Dentistry, NCRP Report No. 145.

26. National Council on Radiation Protection and Measurements. Reference Levels and Achievable Doses in Medical and Dental Imaging: Recommendations for the United States. NCRP Report No. 172.

27. International Commission on Radiological Protection. Radiological protection 
and safety in medicine, ICRP report 73. Ann ICRP. 2004:34.

28. International Commission on Radiological Protection. Managing patient dose in digital radiology, ICRP Publication 93. Ann ICRP 2004: 34.

29. Nishikawa K, Ooguro T, Kuroyanagi K. Comparisons of physical imaging properties among three kinds of imaging plates used in photostimulable phosphor systems for dental radiography. Bull Tokyo Dent Coll 2002;43:23-30.

30. Doyle P, Finney L. Performance evaluation and testing of digital intra-oral radiographic systems. Radiat Protect Dosimet 2005;117:313-7.

31. Udupa H, Mah P, Dove SB, McDavid WD. Evaluation of image quality parameters of representative intraoral digital radiographic systems. Oral Surg Oral Med Oral Pathol Oral Radiol 2013;116: 774-83.

32. Bhaskaran V, Qualtrough AJ, Rushton VE, Worthington HV, Horner K. A laboratory comparison of three imaging systems for image quality and radiation exposure characteristics. Int Endod J 2005;38: 645-52. 
Captions to the tables and legends:

Tables:

Table 1. Technical specifications of the imaging systems.

Table 2. Mean radiopacity values and standard deviations of CMOS and PSP groups in shooting tracts.

Table 3. Distribution of the CMOS and PSP groups in regard to EgAl.

Figures:

Fig. 1 Alignment of the test material on PSP. 
Tables:

Table 1. Technical specifications of the imaging systems.

$\begin{array}{llll}\text { System } & \text { Receptor Technology } & \begin{array}{l}\text { Active surface } \\ \text { dimension }(\mathrm{mm})\end{array} & \text { Pixel size }(\mathrm{mm}) \\ \text { CS 5100 } & \begin{array}{l}\text { RVG sensor technology with } \\ \text { optical fiber (CMOS) }\end{array} & 14 \mathrm{lp} / \mathrm{mm} \\ \text { Sopro PSP } & \begin{array}{l}\text { Photostimulable Phosphor } \\ \text { Plate System }\end{array} & 24 \times 40 & 14 \mathrm{lp} / \mathrm{mm} \\ & & & \\ & & \end{array}$

Table 2. Mean radiopacity values and standard deviations of CMOS and PSP groups in shooting tracts.

\begin{tabular}{lllll}
\hline & CMOS & & PSP & \\
\cline { 2 - 5 } & Mean & SD & Mean & SD \\
\hline $\mathbf{0 . 0 8 ~ s e c}$ & 181 & 14.68 & 238.12 & 7.14 \\
$\mathbf{0 . 1} \mathbf{~ s e c}$ & 180.22 & 14.76 & 230.42 & 12.6 \\
$\mathbf{0 . 1 2 5} \mathbf{~ s e c}$ & 180.47 & 14.5 & 213.42 & 15.53 \\
$\mathbf{0 . 1 6} \mathbf{~ s e c}$ & 178.46 & 15.75 & 188.62 & 19.2 \\
\hline
\end{tabular}


Table 3. Distribution of the CMOS and PSP groups in regard to EgAl.

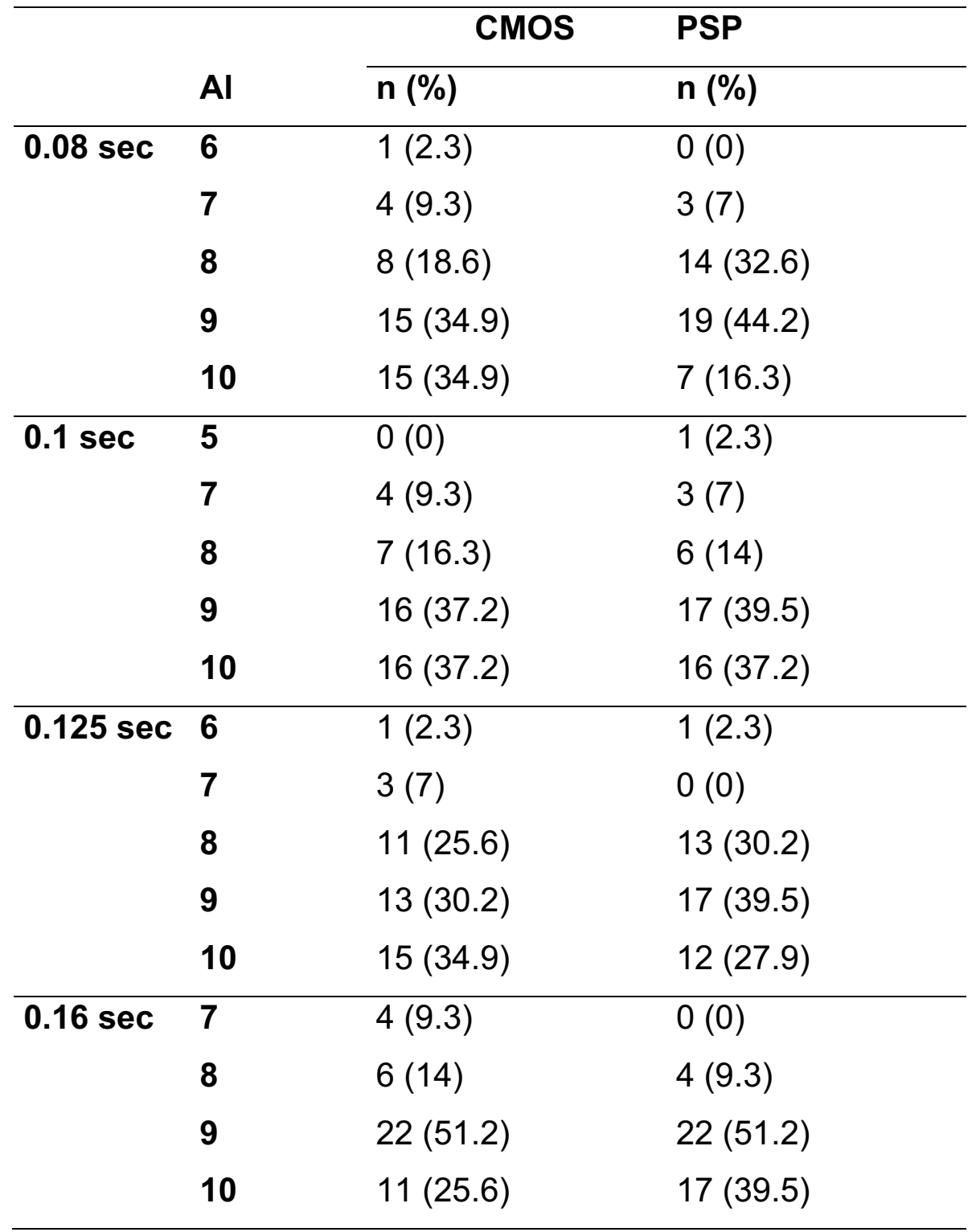




\section{Figures:}

Fig. 1 Alignment of the test material on PSP.

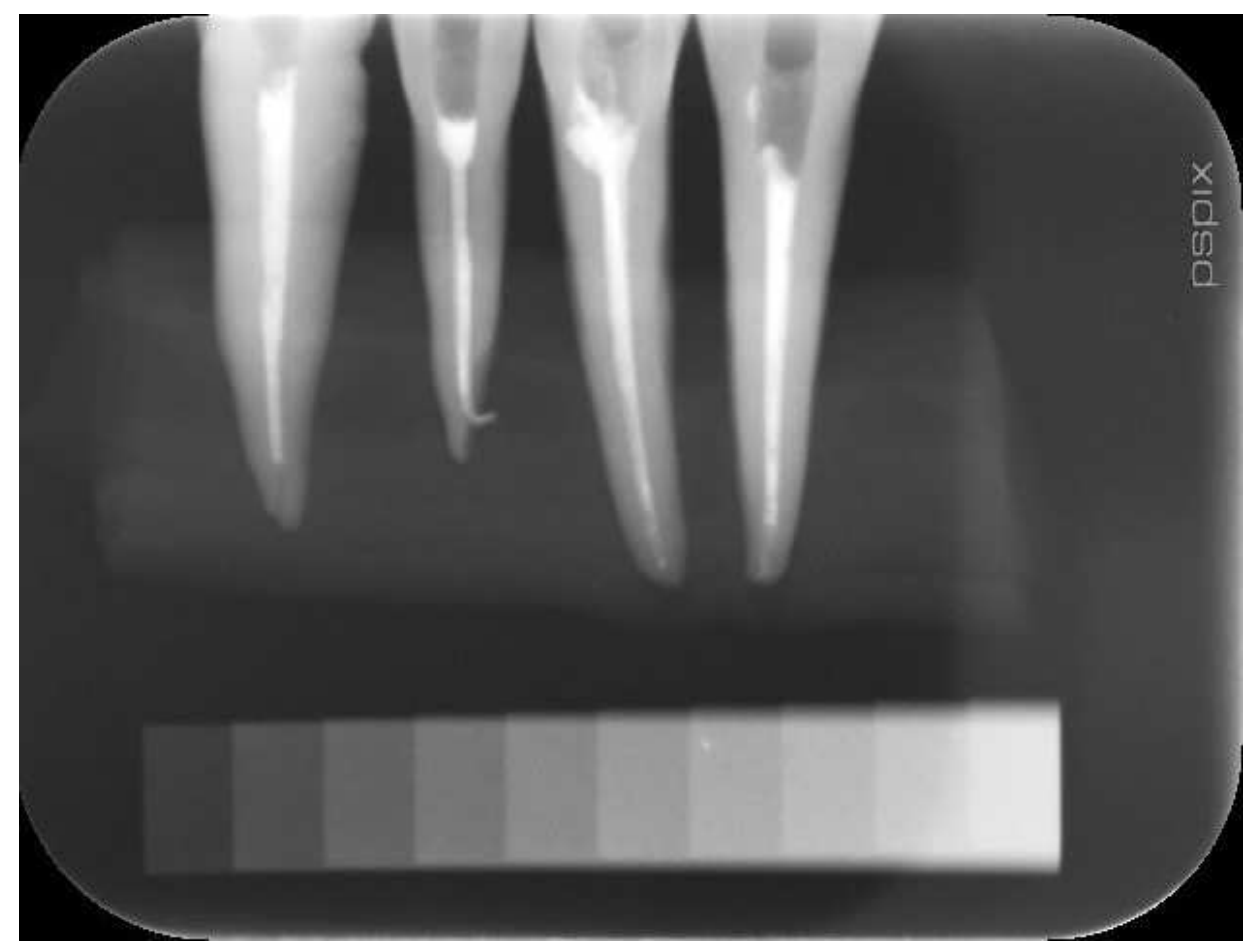

